\title{
Development of minimal basic data set to report COVID-19
}

\author{
Mostafa Shanbehzadeh ${ }^{1}$, Hadi Kazemi-Arpanahi*2 (1) \\ Received: 13 May 2020 \\ Published: 1 Sep 2020
}

\begin{abstract}
Background: Effective surveillance of COVID-19 highlights the importance of rapid, valid, and standardized information to crisis monitoring and prompts clinical interventions. Minimal basic data set (MBDS) is a set of metrics to be collated in a standard approach to allow aggregated use of data for clinical purposes and research. Data standardization enables accurate comparability of collected data, and accordingly, enhanced generalization of findings. The aim of this study is to establish a core set of data to characterize COVID-19 to consolidate clinical practice.

Methods: A 3-step sequential approach was used in this study: (1) an elementary list of data were collected from the existing information systems and data sets; (2) a systematic literature review was conducted to extract evidence supporting the development of MBDS; and (3) a 2-round Delphi survey was done for reaching consensus on data elements to include in COVID-19 MBDS and for its robust validation.

Results: In total, 643 studies were identified, of which 38 met the inclusion criteria, where a total of 149 items were identified in the data sources. The data elements were classified by 3 experts and validated via a 2-round Delphi procedure. Finally, 125 data elements were confirmed as the MBDS.

Conclusion: The development of COVID-19 MBDS could provide a basis for meaningful evaluations, reporting, and benchmarking COVID-19 disease across regions and countries. It could also provide scientific collaboration for care providers in the field, which may lead to improved quality of documentation, clinical care, and research outcomes.
\end{abstract}

Keywords: Minimum basic data set, Minimum data set, COVID-19

Conflicts of Interest: None declared

Funding: This article is extracted from a research project supported by Abadan Faculty of Medical Sciences (IR.ABADANUMS.REC.1399.021). We also thank the Research Deputy of Abadan faculty of Medical Sciences for financially supporting this project

*This work has been published under CC BY-NC-SA 1.0 license.

Copyright $\odot$ Iran University of Medical Sciences

Cite this article as: Shanbehzadeh M, Kazemi-Arpanahi H. Development of minimal basic data set to report COVID-19. Med J Islam Repub Iran. 2020 (1 Sep);34:111. https://doi.org/10.47176/mjiri.34.111

\section{Introduction}

In December 2019, a series of cases of pneumonia with mysterious etiology was first identified in Wuhan, China. On January 7, 2020, the novel Coronavirus (COVID-19),

Corresponding author: Dr Hadi Kazemi-Arpanahi, h.kazemi@abadanums.ac.ir

1. Department of Health Information Technology, School of Paramedical, Ilam University of Medical Sciences, Ilam, Iran

2. Department of Health Information Technology, Abadan Faculty of Medical Sciences, Abadan, Iran previously known as Severe Acute Respiratory Syndrome Coronavirus2 (SARS-CoV-2 or 2019-nCoV) was identified as the causal organism (1-3). COVID-19 is classified

\section{$\uparrow$ What is "already known" in this topic:}

There is no established national core data set for the COVID19 in Iran, which has led to a lack of standardization and variable assessment criteria being used across the country. This hinders the quality and monitoring of COVID-19.

\section{$\rightarrow$ What this article adds:}

Using a structured approach, we developed a minimal dataset to underpin COVID-19 documentation and practice. It is anticipated that the MBDS will facilitate a more consistent approach to COVID-19 practice. This dataset can also be used by other researchers to apply statistical analyses and machine learning algorithms to compare the characteristics of the pandemic among different countries and to identify characteristics that could bring new insights about the pandemic and how to fight it. 
as a type of RNA virus, belonging to the family of coronaviruses, which primarily leads to a respiratory system infection and is extensively transmitted among humans and mammals, causing numerous conditions that range from the "common" influenza to death $(4,5)$. COVID-19 seems to be extremely communicable. The World Health Organization (WHO) has recently confirmed the COVID19 a public health emergency (6). The WHO is warning countries to expand their efforts to contain the disease and safeguard health care environments and notes that a solution calls for a worldwide 'aggressive preparedness' (7).

Early, systematic, and active emergency management practices are key points in epidemic prevention and control. The effective surveillance of this emerging outbreak heavily relies on regulatory management and coordinated interventions, which include comprehensive and directed surveillance, antimicrobial stewardship program, education and training, research and epidemiological studies, and policymaking, etc. These interventions highlight the importance of rapid, valid, and reliable information sharing across hospitals and public health authorities for monitoring crisis and early warning. In this situation, highquality datasets are the prerequisite of necessary analysis for public health, which is inherently a data-intensive domain (8-12). In Iran, most organizations have developed different processes and infrastructure for management and subsequent data collection of COVID-19 patients (13-17). Although current efforts to report COVID-19 are a good start, the absence of information management viewpoint regarding which data elements are critical to be recorded leads to significant inconsistent, unreliable, redundant, or duplicate reports. Thus, this precludes data integration, which limits the share of data across multiple health information systems $(18,19)$.

Further, standardized clinical documentation is an essential factor for electronic health records (EHRs) and for supporting secondary use of data gathered in the context of clinical daily workflows for other purposes than patient care, eg, for clinical research, quality management, epidemiologic studies, patient outcomes, and interoperability initiatives. MBDS is a data collection tool that aims to identify the common components of data sets as one of the first and most basic steps in foundation and implementation of numerous information systems through minimizing duplication of effort and improving data quality (20-24). COVID-19 monitoring depends on clinical data and reports from widely scattered public and hospital information systems as data input (eg, Hospital information systems (HIS), Iranian Electronic Health Record (socalled SEPAS), Iranian Integrated Health System (known as SIB), and other clinical information systems). Accordingly, as we are in the primary step of this emergency, the need to establish a supportive, standardized, accurate, and updated dataset is of paramount importance. Adopting such dataset is an important step in promoting data (capture) and data exchange with regard to COVID-19. Thus, we conducted a systematic literature review combined with a Delphi survey to establish a minimal dataset that would be regarded as a standardized method of reporting COVID-19 disease, and thus it is expected to improve the quality of clinical and research outcomes.

\section{Methods}

Design

In this study, a 3-step sequential approach was used. First, an elementary list of data was collected from the existing information systems and datasets. Next, a search strategy was developed to identify data items for establishing COVID-19 MBDS from an evidence-based perspective. These sources were continuously reviewed until data saturation (maximum data set). Finally, the data included from the review were analyzed using a 2-round Delphi survey to achieve consensus on optimal data set (minimum data set).

\section{Data collection}

The initial data elements were extracted from the medical records of patients with COVID-19, reports from Corona National headquarters, and other clinical and public health organizations affiliated to the Iranian Ministry of Health as well as official dataset provided by international organizations, such as the World Health Organization (WHO) reports, European Centre for Disease Prevention and Control dataset (ECDC), Chinese Center for Disease Control and Prevention (Chinese CDC). In addition to mapping available evidence supporting the development of minimal dataset, a systematic review was also conducted to identify probable data elements for inclusion in COVID-19 MBDS. To that end, PubMed, Scopus, Web of Science, and Google Scholar databases were reviewed by the following search terms (designed using English MeSH keywords and Emtree terms): "COVID-19", "Novel coronavirus 2019", "2019 nCoV”, "clinical characteristics", "clinical features" and "clinical findings". In Table 1, the systematic search strategy is proposed based on Boolean search operators, keywords, and search fields (advance

Table 1. Search strategy details

\begin{tabular}{ll}
\hline Databases & Search details \\
\hline PubMed & $((((((()(((($ covid-19 "[Title]) OR "novel coronavirus"[Title]) OR "2019 nCoV"[Title]) AND clinical characteristics ([Title/ \\
& Abstract]) OR clinical features [Title/ Abstract]) (English [lang]), limited to 2019-2020. \\
Scopus & (TITLE (covid-19) OR TITLE (novel AND coronavirus) OR TITLE (2019 nCoV) AND TITLE-ABS-KEY (clinical AND \\
& features) OR TITLE-ABS-KEY (clinical AND characteristics)) AND (LIMIT-TO (PUBSTAGE, "final")) AND (LIMIT- \\
& TO ( PUBYEAR, 2020) OR LIMIT-TO ( PUBYEAR, 2019)) AND (LIMIT-TO (DOCTYPE, "ar")) AND (LIMIT-TO \\
& (LANGUAGE, "English")). \\
Web of Science & TITLE: (COVID-19) OR TITLE: (novel coronavirus) OR TITLE: (2019 nCoV) AND TOPIC: (clinical findings) OR TOPIC: \\
& (clinical characteristics(Refined by: DOCUMENT TYPES: (ARTICLE) AND PUBLICATION YEARS: ( 2019-2020)) \\
Scholar & allintitle: (COVID-19 OR "novel coronavirus") AND ("clinical features" OR "clinical characteristics") AND English [lang], \\
& limited to 2019-2020.
\end{tabular}


search interface).

\section{Data material}

Two authors independently performed electronic literature searches for study identification and screening. The results of the initial search strategy were first screened based on the title and abstract. The full-texts of relevant articles were examined for inclusion and exclusion criteria. This research included all full-text articles extracted from reliable sources in English between December 2019 and April 2020. Short articles, letters to the editor, accepted papers in conferences, thesis, and reports extracted from blogs were not included in this study. The main criterion for selection of research articles was the relevancy of their content with the research title. Due to the large number of available research articles, several criteria were considered for selecting articles and introducing clinical core data elements to report COVID-19. Hence, full articles with at least 2 of the following data classes related to the main objectives of reporting of COVID-19 were selected: (1) clinical, (2) laboratory, (3) radiology, and (4) epidemiological features. Finally, probable data elements to be included in COVID-19 MBDS were introduced in a checklist.

\section{Questionnaire development}

A questionnaire was developed using the data elements of the checklist and included 5 columns: "very important", "important" "neutral", "slightly important", and "very slightly important" for each data item. To add necessary data elements by experts, a blank row was provided at the end of the questionnaire. The content validity of the questionnaire was assessed by an expert panel, including 2 infectious specialists and 3 health information management (HIM) experts. To add necessary data elements by experts, a blank row was provided at the end of the questionnaire. Test-retest (at an 8-day interval) was done to determine the reliability of the questionnaires, based on experts' answers, including 2 health information management (HIM) and 2 medical informatics experts. Finally, the collected data were analyzed using SPSS 16, with the questionnaire showing a Cronbach's alpha of 0.86 .

\section{Delphi phase}

The data elements were validated using 2 rounds of the Delphi survey by a group of multidisciplinary medical experts (Table 2). The experts participating in the study were asked to score the tabulated list of data elements in terms of their importance using a 5-point Likert scale (ranging from 1:"very slightly important" to 5:"highly important". The level of agreement was considered to be a criterion for the acceptance of the data elements. Thus, after initial ranking, data elements with $\leq 50 \%$ agreement were excluded in the first round, those with 50\%-75\% agreement entered the second round, and data elements with $\geq 75 \%$ agreement were included in the primary round.

\section{Results}

A total of 643 articles were obtained from the literature review. After removal of duplicate articles and applying the exclusion criteria, 38 articles were included in the analysis (Fig. 1).

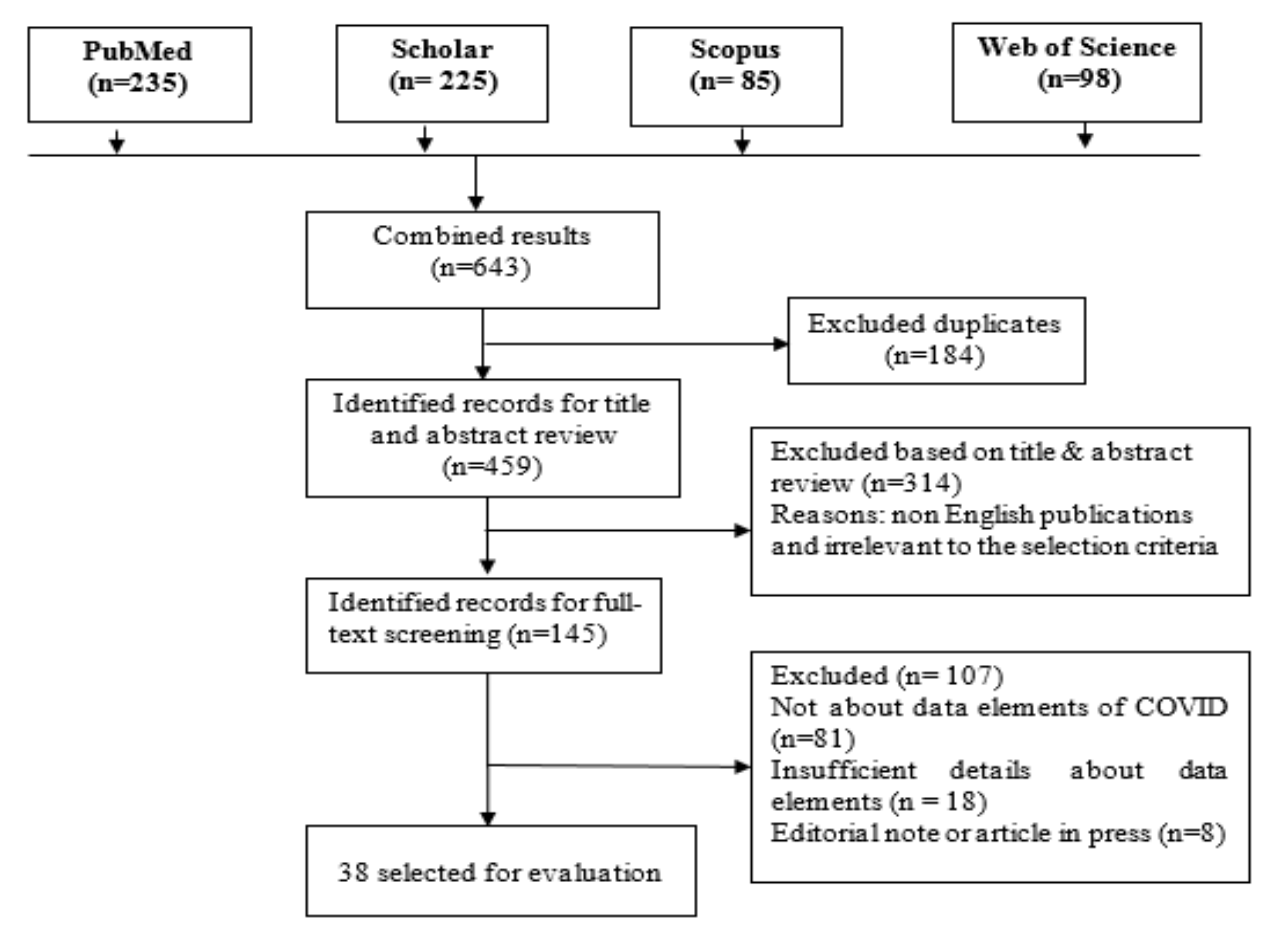

Fig. 1. Flow diagram showing publication selection process 


\begin{tabular}{|c|c|c|}
\hline Variables & Frequency & Percentage \\
\hline \multicolumn{3}{|l|}{ Specialty } \\
\hline Infectious disease & 6 & 31.58 \\
\hline Internal medicine & 5 & 26.31 \\
\hline Radiologist & 4 & 21.05 \\
\hline Epidemiologist & 4 & 21.05 \\
\hline \multicolumn{3}{|l|}{ Gender } \\
\hline Female & 6 & 31.58 \\
\hline Male & 13 & 68.42 \\
\hline \multicolumn{3}{|l|}{ Age (years) } \\
\hline $30-40$ & 6 & 31.58 \\
\hline $40-50$ & 7 & 36.84 \\
\hline $50-60$ & 5 & 26.31 \\
\hline$>60$ & 1 & 5.27 \\
\hline \multicolumn{3}{|l|}{ Work experience (years) } \\
\hline$<10$ & 7 & 36.84 \\
\hline $10-20$ & 8 & 42.10 \\
\hline $20-30$ & 3 & 15.78 \\
\hline$>30$ & 1 & 5.27 \\
\hline Total & 19 & 100 \\
\hline
\end{tabular}

The demographic data of the study participants are presented in Table 2. The potential participants consisted of 25 medical specialists involved in COVID-19 care, treatment, and research domains. However, 6 specialists did not participate in the study. Thus, 19 experts contributed.

Overall, 3 data categories, 19 data classes and 149 data items were extracted from the related comprehensive literature review (maximum dataset). These data categories were epidemiological, clinical, and paraclinical. Epidemiological data contained 4 categories, including basic in- formation, exposure history, transmission mode, and susceptible populations. The clinical data category consisted of clinical manifestations, coexisting conditions, treatment and supportive care, physical examinations, complications, time intervals, disease severity, disease status, and outcome data classes. Finally, the paraclinical category was divided into 2 laboratory and radiology indicators. The definitive numbers of data elements for epidemiological, clinical and paraclinical classes were 25,73 , and 51 respectively (Table 3 ).

\begin{tabular}{|c|c|c|c|c|c|}
\hline Literature review results & \multirow[b]{2}{*}{ Frequency } & \multicolumn{4}{|c|}{$\begin{array}{l}\text { Specialists perspectives } \\
\checkmark \\
: \text { : Accepted in first round } \\
\times: \text { Rejected in first round } \\
\circ: \text { Refer to second round }\end{array}$} \\
\hline Data classes / items & & $\begin{array}{l}\text { First round } \\
\text { Mean } \\
\text { (percentage) }\end{array}$ & $\begin{array}{c}\text { Initial } \\
\text { decision }\end{array}$ & $\begin{array}{c}\text { Second round } \\
\text { Mean } \\
\text { (percentage) }\end{array}$ & $\begin{array}{c}\text { Final } \\
\text { decision }\end{array}$ \\
\hline \multicolumn{6}{|l|}{ A. Epidemiological data } \\
\hline Age $(2-4,25-44)$ & 23 & $4.26(85.2)$ & $\checkmark$ & & Accept \\
\hline $\operatorname{Sex}(2-4,25,27-29,31-44)$ & 21 & $3.90(78)$ & $\checkmark$ & & Accept \\
\hline Occupation $(40,42)$ & 2 & $2.1(42)$ & $x$ & & Refuse \\
\hline Nationality / race $(40,42)$ & 2 & $3.37(67.36)$ & $\circ$ & $3.32(66.31)$ & Refuse \\
\hline \multicolumn{6}{|l|}{ Exposure information } \\
\hline Exposure history $(33,35,36,41,42,45)$ & 6 & $4.52(90.52)$ & $\checkmark$ & & Accept \\
\hline Uncertainty $(32,36,39,46)$ & 4 & $4.32(86.31)$ & $\checkmark$ & & Accept \\
\hline Living in epidemic area $(2,4,32-34,46)$ & 6 & $4.21(84.21)$ & $\checkmark$ & & Accept \\
\hline Recent travelling $(2,4,32,34,37,42,44)$ & 7 & $4.26(85.20)$ & $\checkmark$ & & Accept \\
\hline Contact with suspicious person $(2,4,32-34,46)$ & 6 & $4.36(87.36)$ & $\checkmark$ & & Accept \\
\hline \multicolumn{6}{|l|}{ Transmission mode } \\
\hline Person-person $(2,33-35,42,46-48)$ & 7 & $3.9(77.90)$ & $\checkmark$ & & Accept \\
\hline Nosocomial $(34,35,42,46,48)$ & 5 & $4.26(85.2)$ & $\checkmark$ & & Accept \\
\hline Inhalation (aerosols) $(35,42,48)$ & 3 & $3.32(66.32)$ & o & $2.79(55.79)$ & Refuse \\
\hline Food / water born $(35,42,48)$ & 3 & $3.37(67.36)$ & o & $1.87(37.40)$ & Refuse \\
\hline Contaminated surfaces $(33,35,42,47)$ & 4 & $4.16(83.15)$ & $\checkmark$ & & Accept \\
\hline Sporadic occurrence of zoonotic $(35,42)$ & 2 & $1.07(21.4)$ & $x$ & & Refuse \\
\hline Other $(35,42)$ & 2 & $3.95(78.95)$ & $x$ & & Accept \\
\hline Susceptible population & 2 & $3.95(78.95)$ & $\checkmark$ & & Accept \\
\hline Elderly people $(32,36,39,46)$ & 4 & $4.47(89.48)$ & $\checkmark$ & & Accept \\
\hline Current pregnancy $(32,36,40,45)$ & 4 & $4.53(90.53)$ & $\checkmark$ & & Accept \\
\hline Poor immune function $(32,40,45)$ & 3 & $3.84(76.84)$ & $\checkmark$ & & Accept \\
\hline
\end{tabular}


M. Shanbehzadeh, et al.

\begin{tabular}{|c|c|c|c|c|c|}
\hline \multirow{2}{*}{$\begin{array}{l}\text { Literature review results } \\
\text { Data classes / items }\end{array}$} & \multirow[b]{2}{*}{ Frequency } & \multicolumn{4}{|c|}{$\begin{array}{l}\text { Specialists perspectives } \\
\checkmark \text { : Accepted in first round } \\
\times: \text { Rejected in first round } \\
\circ \text { : Refer to second round }\end{array}$} \\
\hline & & $\begin{array}{l}\text { First round } \\
\text { Mean } \\
\text { (percentage) } \\
\end{array}$ & $\begin{array}{c}\text { Initial } \\
\text { decision }\end{array}$ & $\begin{array}{c}\text { Second round } \\
\text { Mean } \\
\text { (percentage) } \\
\end{array}$ & $\begin{array}{c}\text { Final } \\
\text { decision }\end{array}$ \\
\hline A. Epidemiological data & & & & & \\
\hline Susceptible population & 2 & $3.95(78.95)$ & $\checkmark$ & & Accept \\
\hline Chronic co-morbidities $(32,36,46)$ & 3 & $3.79(75.8)$ & $\checkmark$ & & Accept \\
\hline Long-term use of immunosuppressive $(32,40)$ & 2 & $4.26(85.2)$ & $\checkmark$ & & Accept \\
\hline Surgery history $(32,40)$ & 2 & $2.79(55.79)$ & 0 & $3.32(66.31)$ & Refuse \\
\hline Active smoker $(32,39,40)$ & 3 & $4.37(87.37)$ & $\checkmark$ & & Accept \\
\hline Other $(32,40)$ & 2 & $3.95(78.95)$ & $\checkmark$ & & Accept \\
\hline \multicolumn{6}{|l|}{$\begin{array}{l}\text { B. Clinical data } \\
\text { Clinical manifestations }\end{array}$} \\
\hline Fever $(2-4,25-27,29-37,39-45,47-54)$ & 29 & $4.53(90.53)$ & $\checkmark$ & & Accept \\
\hline Dry cough $(2-4,25,26,29-37,39-43,45,47-54)$ & 27 & $4.37(87.37)$ & $\checkmark$ & & Accept \\
\hline Sputum / expectoration $(2-4,26,29,31,34,39,40,43,44,48-53)$ & 16 & $3.58(71.57)$ & ○ & $3.98(79.6)$ & Accept \\
\hline Dyspnea $(2-4,25-27,29-37,39,40,42-45,47-54)$ & 27 & $4.26(85.2)$ & $\checkmark$ & & Accept \\
\hline Myalgia or fatigue $(2-4,25-27,29,31-34,36,39,41-44,48-54)$ & 22 & $4.05(81)$ & $\checkmark$ & & Accept \\
\hline Headache $(2-4,25,26,29,30,32-36,39,42-44,48-52)$ & 20 & $2.47(49.47)$ & $\times$ & & Refuse \\
\hline Sore throat $(2-4,25,26,29,31,32,34-36,39,40,42-44,48-53)$ & 21 & $3.84(76.84)$ & $\checkmark$ & & Accept \\
\hline Dizziness $(2,3,26,37,40,43,44,48,50)$ & 9 & $2.42(48.42)$ & $x$ & & Refuse \\
\hline Rhinorrhea $(2,3,26,32,40,43,44,48,49,52)$ & 10 & $3.53(70.53)$ & $\circ$ & $3.95(79)$ & Accept \\
\hline Chest pain $(2,3,25,26,32,34,35,40,42-44,48,49,54)$ & 14 & $2.47(49.47)$ & $x$ & & Refuse \\
\hline Pharyngeal congestion $(30,34,36,43,44,48,52,53)$ & 8 & $3.78(75.79)$ & $\checkmark$ & & Accept \\
\hline Chill $(2-4,34,44,48)$ & 6 & $3.35(67)$ & o & $3.65(73)$ & Refuse \\
\hline Abdominal pain $(29,31,32,34,39,42,43,48,54)$ & 9 & $2.37(47.37)$ & $x$ & & Refuse \\
\hline Diarrhea $(2-4,25-27,29-33,36,37,39,40,43,44,48-52,54)$ & 23 & $2.15(43.16)$ & $x$ & & Refuse \\
\hline Anorexia $(2-4,26,32,36,42,44,48-50,52)$ & 12 & $2.73(54.73)$ & o & $2.87(57.4)$ & Refuse \\
\hline Vomiting and nuusea $(4,29-33,35,39,42-44,48,52,53)$ & 14 & $3.15(63)$ & ○ & $3.48(69.60)$ & Refuse \\
\hline More than one sign or symptom $(26,34,36,48)$ & 4 & $4.58(91.59)$ & $\checkmark$ & & Accept \\
\hline No sign or symptom (asymptomatic) $(42,47,48,52)$ & 4 & $4.16(83.16)$ & $\checkmark$ & & Accept \\
\hline \multicolumn{6}{|l|}{ Co-existing conditions } \\
\hline $\begin{array}{l}\text { Hypertension }(3,25,26,28,29,31,32,34,36,38-40,42-44,48-50, \\
54,55)\end{array}$ & 20 & $3.84(76.84)$ & $\checkmark$ & & Accept \\
\hline $\begin{array}{l}\text { Cardiovascular }(3,25-29,31,32,34,36,38-40,42,43,48-50,54 \text {, } \\
55)\end{array}$ & 20 & $4.32(86.32)$ & $\checkmark$ & & Accept \\
\hline Cerebrovascular $(3,25,26,40,43,49,50,54,55)$ & 9 & $4.05(81.05)$ & $\checkmark$ & & Accept \\
\hline Diabetes $(3,25-29,32,34,36,38,43,44,49,50,53-55)$ & 17 & $3.84(76.84)$ & $\checkmark$ & & Accept \\
\hline Gastrointestinal disease $(26,36,50,51)$ & 4 & $3.26(65.26)$ & $\circ$ & $3.97(79.40)$ & Accept \\
\hline Malignant tumors $(3,25,26,28,31,34,38,43,49,50,54,55)$ & 12 & $4.05(81.05)$ & $\checkmark$ & & Accept \\
\hline Neural system disease $(25,26,29,34,39,40,43,49,50,53,54)$ & 11 & $3.05(61.05)$ & ० & $3.63(72.63)$ & Refuse \\
\hline Pulmonary disease $(25,26,28,31,34,38,43,49,50,53,54)$ & 12 & $4.84(96.84)$ & $\checkmark$ & & Accept \\
\hline Chronic liver disease $(28,29,34,38,40,43,50,53-55)$ & 11 & $4.53(90.53)$ & $\checkmark$ & & Accept \\
\hline Chronic kidney disease $(28,29,34,38-40,42,43,50,53-55)$ & 12 & $3.79(75.8)$ & $\checkmark$ & & Accept \\
\hline HIV / immunodeficiency $(26,39,43,50,53)$ & 5 & $3.84(76.84)$ & $\checkmark$ & & Accept \\
\hline Virus - bacterial coinfection $(26,36,50,51)$ & 4 & $3.84(76.84)$ & $\checkmark$ & & Accept \\
\hline None $(28,39)$ & 2 & $3.79(75.8)$ & $\checkmark$ & & Accept \\
\hline \multicolumn{6}{|l|}{ Treatment \& supportive } \\
\hline \multicolumn{6}{|l|}{ Mechanical ventilation } \\
\hline Non-invasive (NMV) $(4,26,30,34,36,38,40-42,48,51-53)$ & 13 & $4.11(82.10)$ & $\checkmark$ & & Accept \\
\hline Invasive (IMV) $(4,26,30,34,36,38,40,41,44,48,51-53)$ & 13 & $4.21(84.21)$ & $\checkmark$ & & Accept \\
\hline Extracorporeal Membrane (ECMO) $(4,26,30,34,38,40,51,52)$ & 8 & $4(80)$ & $\checkmark$ & & Accept \\
\hline Both ECMO and IMV $(30,42,51)$ & 3 & $3.84(76.84)$ & $\checkmark$ & & Accept \\
\hline \multicolumn{6}{|l|}{ Prescription } \\
\hline Antibiotic $(4,26,34,36,38,40,41,44,48,49,52,53)$ & 12 & $3.79(75.8)$ & $\checkmark$ & & Accept \\
\hline Antifungal $(4,26,34,36,42,44,48,49,52,53)$ & 10 & $1.84(36.84)$ & $\times$ & & Refuse \\
\hline Antiviral $(4,26,34,40,42,44,48,49,51-53)$ & 11 & $4.21(84.21)$ & $\checkmark$ & & Accept \\
\hline Glucocorticoids $(4,26,34,36,38,44,49,51)$ & 8 & $3.79(75.8)$ & $\checkmark$ & & Accept \\
\hline Immunoglobulin $(4,26,34,38,44,49,52)$ & 7 & $4.26(85.2)$ & $\checkmark$ & & Accept \\
\hline
\end{tabular}

Finally, 149 primary data elements were included in the Delphi survey, of which 112 data elements were finalized in the first round and 15 were rejected. A total of 22 data elements progressed to the second round of the Delphi survey. Of them, 9 were approved in round 2. Thus, on completion of the survey, 125 data elements were approved. Accordingly, the final data elements for epidemiological, clinical, and paraclinical categories were 22, 57, 


\begin{tabular}{|c|c|c|c|c|c|}
\hline \multicolumn{6}{|l|}{ Table 3. Ctd } \\
\hline \multicolumn{2}{|l|}{ Literature review results } & \multicolumn{4}{|c|}{$\begin{array}{l}\text { Specialists perspectives } \\
\checkmark: \text { Accepted in first round } \\
\times: \text { Rejected in first round } \\
\circ: \text { Refer to second round } \\
\end{array}$} \\
\hline Data classes / items & Frequency & \multirow[t]{2}{*}{$\begin{array}{c}\text { First round } \\
\text { Mean } \\
\text { (percentage) } \\
\end{array}$} & $\begin{array}{c}\text { Initial } \\
\text { decision }\end{array}$ & $\begin{array}{c}\text { Second round } \\
\text { Mean } \\
\text { (percentage) }\end{array}$ & $\begin{array}{c}\text { Final } \\
\text { decision }\end{array}$ \\
\hline \multicolumn{5}{|l|}{ B. Clinical data } & \\
\hline \multicolumn{6}{|l|}{ Physical examination } \\
\hline Body temperature $(25,26,29,32,34,36,42,45,51-53)$ & 10 & $4.11(82.11)$ & $\checkmark$ & & Accept \\
\hline Respiratory rate (per minute) $(32,34,36,51)$ & 4 & $3.95(78.95)$ & $\checkmark$ & & Accept \\
\hline Heart rate (beats/ per minute) $(32,34,36,51)$ & 4 & $3.84(76.84)$ & $\checkmark$ & & Accept \\
\hline Body Mass Index (BMI) $(36,51)$ & 2 & $2.42(48.42)$ & $\times$ & & Refuse \\
\hline Systolic blood pressure $[\mathrm{mmHg}](32,34,36,51)$ & 4 & $3.95(78.95)$ & $\checkmark$ & & Accept \\
\hline Lung auscultation (sounds) $(34,36,51)$ & 3 & $2.47(49.47)$ & $\times$ & & Refuse \\
\hline \multicolumn{6}{|l|}{ Disease Complication } \\
\hline ARDS $(31,34,36,38,39,41,43,49,51-53,55)$ & 12 & $4(80)$ & $\checkmark$ & & Accept \\
\hline Acute heart injury $(31,34,36,38,41,43,49,51,52)$ & 9 & $4.26(85.2)$ & $\checkmark$ & & Accept \\
\hline Liver abnormality $(34,36,38,39,43,49,51,52)$ & 8 & $3.42(68.42)$ & ○ & $4.05(81.05)$ & Accept \\
\hline Acute kidney injury $(31,36,38,41,43,49,51-53,55)$ & 10 & $4.05(81.05)$ & $\checkmark$ & & Accept \\
\hline Secondary infection $(31,34,36,38,43,51,52)$ & 7 & $3.89(77.89)$ & $\checkmark$ & & Accept \\
\hline Shock $(31,34,36,38,41,43,49,51-53,55)$ & 11 & $4.32(86.31)$ & $\checkmark$ & & Accept \\
\hline Other $(31,34,36,38,43,51,52)$ & 7 & $3.89(77.89)$ & $\checkmark$ & & Accept \\
\hline \multicolumn{6}{|l|}{ Disease severity } \\
\hline Mild $(27,28,30,32,47,49,50,53)$ & 8 & $3.79(75.8)$ & $\checkmark$ & & Accept \\
\hline Moderate $(27,28,30,32,47,49,50,53)$ & 8 & $3.95(78.95)$ & $\checkmark$ & & Accept \\
\hline Severe $(27,28,30,32,47,49,50,53)$ & 8 & $4(80)$ & $\checkmark$ & & Accept \\
\hline Critical $(27,28,30,32,47,49,50,53)$ & 8 & $3.84(76.84)$ & $\checkmark$ & & Accept \\
\hline \multicolumn{6}{|l|}{ Disease status } \\
\hline Active $(32,36,45,46)$ & 4 & $3.89(77.89)$ & $\checkmark$ & & Accept \\
\hline In active $(32,36,45,46)$ & 4 & $4.05(81.05)$ & $\checkmark$ & & Accept \\
\hline Recovered $(32,36,45,46)$ & 4 & $4(80)$ & $\checkmark$ & & Accept \\
\hline \multicolumn{6}{|l|}{ Outcome } \\
\hline Remained in hospital $(4,31,34,36,38,41,47,51,53)$ & 10 & $3.79(75.8)$ & $\checkmark$ & & Accept \\
\hline Healed / discharged $(4,31,34,36,38,41,47,51,53)$ & 9 & $3.84(76.84)$ & $\checkmark$ & & Accept \\
\hline Referred $(32,39,46,52,55)$ & 5 & $3.12(62.40)$ & o & $3.63(72.63)$ & Refuse \\
\hline Critical condition / ICU $(4,31,34,36,38,41,47,51,53)$ & 9 & $4(80)$ & $\checkmark$ & & Accept \\
\hline Death $(4,31,34,36,38,47,51,53,55)$ & 9 & $3.89(77.89)$ & $\checkmark$ & & Accept \\
\hline Partial recovery / follow up $(32,37,39,42,46,49,52,55)$ & 8 & $2.22(44.40)$ & $\times$ & & Refuse \\
\hline \multicolumn{6}{|l|}{ Time interval } \\
\hline Exposure to symptom onset $(3,35-37,39,42)$ & 6 & $4.05(81.05)$ & $\checkmark$ & & Accept \\
\hline Illness to start treatment $(3,35,37,39,42)$ & 5 & $4.19(83.80)$ & $\checkmark$ & & Accept \\
\hline Median incubation period $(29,46,48,49)$ & 4 & $3.79(75.8)$ & $\checkmark$ & & Accept \\
\hline Hospitalization date $(37,42)$ & 2 & $2.37(47.36)$ & $\times$ & & Refuse \\
\hline Diagnosis date $(36,37,39,42)$ & 4 & $4.21(84.21)$ & $\checkmark$ & & Accept \\
\hline Hospital day $(36,51)$ & 2 & $2.31(46.31)$ & $\times$ & & Refuse \\
\hline Discharge date $(3,37,42)$ & 3 & $3.95(78.95)$ & $\checkmark$ & & Accept \\
\hline
\end{tabular}

and 46 , respectively.

\section{Discussion}

This study reports the basic required data items originally derived from studying the COVID-19 patients' medical records, existing official data sets, and through conducting a systematic literature review, and Delphi survey. The aim of this study was to identify a set of parameters believed to be essential and sufficient to assist the uniform reporting of data on COVID-19. Through the designed COVID$19 \mathrm{MBDS}$, it is possible to meet some of the data requirements regarding care practice, leading to reliable framework on which health care experts can base their documentation. These elements give both the clinicians and researchers high-quality data to support diagnosis and analysis, respectively. The resulting MBDS is therefore more likely to be acceptable and practical in clinical prac- tice and biomedical research. It has also the potential to homogenize data capturing among public and medical information systems, so that clinical data on COVID-19 can be merged and compared. In addition, data exchange and interoperability can be enhanced using a proper and reliable data set (60). Development of a required data set is the most fundamental step for construction of any information system in the health care sector. Determining these data elements based on viewpoints and real requirements of their customers or users can help designers and vendors of information systems to facilitate and accelerate the development of such systems and reduce the possibility of their failure (61). Thus, the MBDS established in this study can be used as a basis for developing different information systems for collection and management of COVID-19 data.

In the context of COVID-19, huge volumes of data are 
M. Shanbehzadeh, et al.

\begin{tabular}{|c|c|c|c|c|c|}
\hline Literature review results & \multirow[b]{2}{*}{ Frequency } & \multicolumn{2}{|c|}{$\begin{array}{l}\text { Specialists perspectives } \\
\checkmark: \text { Accepted in first round } \\
\times: \text { Rejected in first round } \\
\circ: \text { Refer to second round }\end{array}$} & \multirow[b]{2}{*}{$\begin{array}{c}\text { Second round } \\
\text { Mean } \\
\text { (percentage) }\end{array}$} & \multirow[b]{2}{*}{$\begin{array}{c}\text { Final } \\
\text { decision }\end{array}$} \\
\hline Data classes / items & & $\begin{array}{l}\text { First round } \\
\text { Mean } \\
\text { (percentage) }\end{array}$ & $\begin{array}{c}\text { Initial } \\
\text { decision }\end{array}$ & & \\
\hline $\begin{array}{l}\text { C. Paraclinical Data } \\
\text { Laboratory indicators } \\
\text { Blood routine tests }\end{array}$ & & & & & \\
\hline $\begin{array}{l}\text { T lymphocyte count } \times 109 / \mathrm{L} \uparrow \downarrow(26,30,32,34,35,38,39,42,43,48,49 \text {, } \\
56)\end{array}$ & 12 & $4.26(85.26)$ & $\checkmark$ & & Accept \\
\hline Platelet count $\times 109 / \mathrm{L} \uparrow \downarrow(26,30,32,34,35,37-39,42,43,48,49,56)$ & 13 & $3.26(65.26)$ & ० & $3.79(75.8)$ & Accept \\
\hline Hemoglobin level (g/L) $\uparrow \downarrow(26,30,32,34,38,39,42,43,48,49,56)$ & 11 & $4.21(84.21)$ & $\checkmark$ & & Accept \\
\hline D-dimer $(\mathrm{ug} / \mathrm{ml}) \uparrow \downarrow(26,29,30,32,34,39,49,56)$ & 9 & $4.16(83.16)$ & $\checkmark$ & & Accept \\
\hline $\begin{array}{l}\text { Prothrombin times, } \mathrm{s} \uparrow \downarrow(29,30,34,42,43,49) \\
\text { Blood chemistry }\end{array}$ & 7 & $3.37(67.37)$ & $\circ$ & $3.32(66.31)$ & Refuse \\
\hline $\operatorname{ALT}(\mathrm{U} / \mathrm{L}) \uparrow \downarrow(26,29,30,32,37,39,42,43,48,49,56)$ & 11 & $4.31(85.31)$ & $\checkmark$ & & Accept \\
\hline AST (U/L) $\uparrow \downarrow(26,29,30,32,37,39,42,43,48,49,56)$ & 12 & $4.42(88.42)$ & $\checkmark$ & & Accept \\
\hline Blood Urea Nitrogen $(\mathrm{mmol} / \mathrm{L}) \uparrow \downarrow(26,37,42,43,48,56)$ & 6 & $4.26(85.2)$ & $\checkmark$ & & Accept \\
\hline Serum creatinine (umol/L) $\uparrow \downarrow(34,39,42,43,48)$ & 5 & $3.84(76.84)$ & $\checkmark$ & & Accept \\
\hline Lactate dehydrogenase $(\mathrm{U} / \mathrm{L}) \uparrow \downarrow(26,29,30,32,34,39,42,49,56)$ & 3 & $3.63(72.63)$ & ○ & $4.26(85.26)$ & Accept \\
\hline Albumin $(\mathrm{g} / \mathrm{L}) \uparrow \downarrow(32,34,42,49,56)$ & 5 & $3.59(71.58)$ & $\circ$ & $3.70(74)$ & Refuse \\
\hline Globulin $(\mathrm{g} / \mathrm{L}) \uparrow \downarrow(32,34,42,49,56)$ & 5 & $3.42(68.42)$ & o & $3.03(60.6)$ & Refuse \\
\hline Total bilirubin(umol/L) $\uparrow \downarrow(26,29,30,32,34,39,42,49,56)$ & 9 & $3.53(70.53)$ & o & $3.68(73.68)$ & Refuse \\
\hline Direct bilirubin(umol/L) $\uparrow \downarrow(4,31,50)$ & 3 & $2.42(48.42)$ & $x$ & & Refuse \\
\hline \multicolumn{6}{|l|}{ Infection-related biomarkers } \\
\hline C-reactive protein (mg/L) $\uparrow \downarrow(26,30,32,37,42,43,48,49,56)$ & 9 & $3.95(78.95)$ & $\checkmark$ & & Accept \\
\hline Procalcitonin $(\mathrm{ng} / \mathrm{ml}) \uparrow \downarrow(26,29,30,32,34,39,42,43,48,49,56)$ & 11 & $2.95(58.95)$ & 0 & $3.11(62.11)$ & Refuse \\
\hline Interleukin $6(\mathrm{pg} / \mathrm{ml}) \uparrow \downarrow(32,34,38,39,42,43,56)$ & 8 & $4.63(92.63)$ & $\checkmark$ & & Accept \\
\hline $\begin{array}{l}\text { RT-PCR }(3,4,26,28-30,32,33,35-38,40-42,44-46,48,49,51-54,57- \\
59)\end{array}$ & 28 & $4.47(89.47)$ & $\checkmark$ & & Accept \\
\hline \multicolumn{6}{|l|}{ Radiology information } \\
\hline Chest X-ray $(41,49,50)$ & 3 & $2.63(52.63)$ & o & $2.75(55)$ & Refuse \\
\hline CT scan $(2-4,25,31,32,40-43,52,58,59)$ & 12 & $4.05(81.05)$ & $\checkmark$ & & Accept \\
\hline Lung ultrasound $(41,50)$ & 2 & $3.52(70.53)$ & ○ & $3.84(76.84)$ & Accept \\
\hline \multicolumn{6}{|l|}{ Pattern of the lesion } \\
\hline Ground glass opacity $(2-4,25,29-32,40-43,45,48,49,52,58,59)$ & 17 & $4.47(89.47)$ & $\checkmark$ & & Accept \\
\hline Consolidation $(2-4,25,32,41-43,45,48,49,52,58,59)$ & 14 & $4.42(88.42)$ & $\checkmark$ & & Accept \\
\hline Both $(2,25,42,45,49,58)$ & 6 & $3.95(78.95)$ & $\checkmark$ & & Accept \\
\hline Patchy shadow $(3,41,42,52,58,59)$ & 6 & $3.84(76.84)$ & $\checkmark$ & & Accept \\
\hline Lymphadenopathy $(3,32,41,42,45,49,58,59)$ & 8 & $3.90(77.9)$ & $\checkmark$ & & Accept \\
\hline Pleural effusion $(32,41,42,45,49,58,59)$ & 7 & $4.15(83.15)$ & $\checkmark$ & & Accept \\
\hline Crazy paving $(32,41,42,45,49,58,59)$ & 7 & $3.95(78.95)$ & $\checkmark$ & & Accept \\
\hline Bronchiectasis $(3,32,42,45,52,58,59)$ & 7 & $4.16(83.15)$ & $\checkmark$ & & Accept \\
\hline Interlobular septal thickening $(3,41,42,45,52,58,59)$ & 7 & $4.05(81.05)$ & $\checkmark$ & & Accept \\
\hline Reticulation $(3,41,42,52,58,59)$ & 6 & $3.68(73.68)$ & ○ & $3.89(77.9)$ & Accept \\
\hline Other $(3,41,52,58)$ & 5 & $3.78(75.79)$ & $\checkmark$ & & \\
\hline Lesion distribution & & & & & Accept \\
\hline Unilateral $(4,25,26,29,31,32,38,42,43,45,48,52,58,59)$ & 14 & $4.42(88.42)$ & $\checkmark$ & & Accept \\
\hline Bilateral $(4,25,26,29,31,32,38,42,43,45,48,52,58,59)$ & 14 & $4.42(88.42)$ & $\checkmark$ & & Accept \\
\hline Lesion morphology & & & & & \\
\hline Patchy / nodular $(25,32,45,47,48,52,58,59)$ & 8 & $4.42(88.42)$ & $\checkmark$ & & Accept \\
\hline Spherical $(25,32,45,47,48,52,58,59)$ & 8 & $3.89(77.9)$ & $\checkmark$ & & Accept \\
\hline Both $(25,32,45,58,59)$ & 5 & $4.10(82.10)$ & $\checkmark$ & & Accept \\
\hline No lesion $(32,45,47,52,58,59)$ & 6 & $3.68(73.69)$ & ○ & $3.95(78.95)$ & Accept \\
\hline
\end{tabular}

generated every day in clinical and public health domains. In such big data area, what can be collected is not an issue; rather attention should be paid to the depth and statistical power of collected data to confirm or disprove a hypothesis, and answer specific questions $(62,63)$. The anticipated hypothesis and questions to be addressed by a health information system or clinical registry should determine the data items that are preferred, and resource accessibility should inform the scope of the data collected to respond to the expected queries. Part of the problem can be due to lack of comparable data derived from limited sharing, unstructured reporting, and lack of standardized data capture strategies $(64,65)$. To resolve this, new advances in data collection instruments improve the fundability, accessibility, interoperability and reusability (FAIR) of data, highlighting the need for uniform data that can be integrated from different fragmented resources (6669). In this regard, the anticipated benefits of the COVID19 MBDS for investigators can include accelerating study initiation, facilitating data exchange and accumulation, and good data management to reach FAIR data. The COVID-19 MBDS aims to facilitate FAIR data collection 


\begin{tabular}{|c|c|c|c|c|c|}
\hline \multirow{2}{*}{$\begin{array}{l}\text { Literature review results } \\
\text { Data classes / items }\end{array}$} & \multirow[b]{2}{*}{ Frequency } & \multicolumn{4}{|c|}{$\begin{array}{l}\text { Specialists perspectives } \\
\checkmark \text { : Accepted in first round } \\
\times: \text { Rejected in first round } \\
\circ: \text { Refer to second round }\end{array}$} \\
\hline & & $\begin{array}{l}\text { First round } \\
\text { Mean } \\
\text { (percentage) }\end{array}$ & $\begin{array}{c}\text { Initial } \\
\text { decision }\end{array}$ & $\begin{array}{c}\text { Second round } \\
\text { Mean } \\
\text { (percentage) }\end{array}$ & $\begin{array}{c}\text { Final } \\
\text { decision }\end{array}$ \\
\hline \multicolumn{6}{|l|}{ C. Paraclinical Data } \\
\hline Early $(30,32,45,52)$ & 4 & $3.84(76.84)$ & $\checkmark$ & & Accept \\
\hline Progressing $(30,32,45,52)$ & 4 & $4.11(82.10)$ & $\checkmark$ & & Accept \\
\hline Severe $(30,32,45,52)$ & 4 & $3.89(77.9)$ & $\checkmark$ & & Accept \\
\hline \multicolumn{6}{|l|}{ Lesion location } \\
\hline Peripheral $(2,25,32,41,45,58,59)$ & 7 & $3.84(76.84)$ & $\checkmark$ & & Accept \\
\hline Central $(2,25,32,41,45,58)$ & 6 & $3.79(75.8)$ & $\checkmark$ & & Accept \\
\hline Both $(2,25,32,58,59)$ & 5 & $4(80)$ & $\checkmark$ & & Accept \\
\hline Random / diffuse $(2,32,45,58)$ & 4 & $3.79(75.8)$ & $\checkmark$ & & Accept \\
\hline \multicolumn{6}{|l|}{ Involved lobe } \\
\hline Right upper lobe $(2,25,32,42,45,58,59)$ & 7 & $4.32(86.31)$ & $\checkmark$ & & Accept \\
\hline Right middle lobe $(2,25,32,42,45,58,59)$ & 7 & $4.05(81.05)$ & $\checkmark$ & & Accept \\
\hline Right lower lobe $(2,25,32,42,45,58,59)$ & 7 & $4.05(81.05)$ & $\checkmark$ & & Accept \\
\hline Left upper lobe $(2,25,32,42,45,58,59)$ & 7 & $3.95(79)$ & $\checkmark$ & & Accept \\
\hline Left lower lobe $(2,25,32,42,45,58,59)$ & 7 & $4.21(84.21)$ & $\checkmark$ & & Accept \\
\hline Peripheral/central $(2,25,32,41,42,45,58,59)$ & 8 & $3.89(77.9)$ & $\checkmark$ & & Accept \\
\hline
\end{tabular}

Peripheral/central $(2,25,32,41,42,45,58,59)$

from COVID-19 individuals with the context of care, evaluation, and research to improve the comparability of data, interdisciplinary communication, and collaboration within the field of COVID-19.

For developing this MBDS, we performed an extensive literature review to identify COVID-19 variables from an evidence-based perspective in a multiresearch study. Then, a 2-round Delphi methodology narrowed down opinions until consensus was reached, during which parameters that may have importance for some applications were excluded from consideration.

This study reported the development of the first MDSCOVID-19 based on state-of-the-art evidence as well as consultation with future users (experts and clinicians). This method could contribute to establishing a balance between scientific theoretical knowledge and technical knowledge as well as applied wisdom from clinical practice to inform the data set. The resulting MBDS is therefore more possible to be satisfactory and practical in clinical practice. We identified the variables required to analyze fundamental aspects, such as transmission patterns, severity, clinical phenotype, prognostic factors, the effectiveness of therapeutic plans and complications, survival estimation, as well as incidence and prevalence of disease across the country.

The literature review only incorporated the search published in the first 4 months of COVID-19 disease during the review period. A more systematic review may have identified additional relevant studies. However, given that the literature review is aimed to identify potential items for inclusion in the MBDS (rather than identifying every paper that considered COVID-19 parameters), and we drew on the collective wisdom of experts in the COVID19 field throughout the consensus process, it seems unlikely that any important aspects of COVID-19 would have remained overlooked.

\section{Conclusion}

To conclude, the developed MBDS used structured agreement methods that integrated a literature review and expert opinion to consolidate COVID-19 documentation, research, and practice. Data collection in line with the configuration presented in this MBDS contributes to unified reporting, probably leading to improved quality of patient documentation, augmented continuity of care, and improved health outcomes regarding COVID-19. COVID19 MBDS is not proposed to be inclusive; it is what the consulted professionals arbitrated to be a manageable, minimal, and essential set that would ideally be provided in all COVID-19-related research studies. This core set can be augmented in each particular project according to the project's purpose and available resources. Future testing in other health care settings is recommended. In the future, further strategies, including a comprehensive search of the literature, should be considered to enhance this MBDS.

\section{Acknowledgments}

This article is extracted from a research project supported by Abadan Faculty of Medical Sciences (IR.ABADANUMS.REC.1399.021). We also thank the Research Deputy of Abadan faculty of Medical Sciences for financially supporting this project. We also would like to thank all experts who participated in this study and played a role in the validation of the data elements.

\section{Conflict of Interests}

The authors declare that they have no competing interests.

\section{References}

1. Peeri NC, Shrestha N, Rahman MS, Zaki R, Tan Z, Bibi S, et al. The SARS, MERS and novel coronavirus (COVID-19) epidemics, the newest and biggest global health threats: what lessons have we learned 
Int J Epidemiol. 2020

2. Long C, Xu H, Shen Q, Zhang X, Fan B, Wang C, et al. Diagnosis of the Coronavirus disease (COVID-19): rRT-PCR or CT? Eur J Radiol. 2020:108961

3.Shi H, Han X, Jiang N, Cao Y, Alwalid O, Gu J, et al. Radiological findings from 81 patients with COVID-19 pneumonia in Wuhan, China: a descriptive study. Lancet Infect Dis. 2020.

4. Yang W, Cao Q, Qin L, Wang X, Cheng Z, Pan A, et al. Clinical characteristics and imaging manifestations of the 2019 novel coronavirus disease (COVID-19): A multi-center study in Wenzhou city, Zhejiang, China. J Infect. 2020.

5. Cruz MP, Santos E, Cervantes MV, Juárez ML. COVID-19, a worldwide public health emergency. Rev Clin Esp. (English Edition). 2020

6. Guan WJ, Ni ZY, Hu Y, Liang WH, Ou CQ, He JX, et al. Clinical characteristics of coronavirus disease 2019 in China. N Engl J Med. 2020

7. Al-Benna S. Availability of COVID-19 Information from National and International Aesthetic Surgery Society Websites. Aesthetic Plast Surg. 2020:1.

8. Idrovo AJ, Manrique-Hernández EF. Data Quality of Chinese Surveillance of COVID-19: Objective Analysis Based on WHO's Situation Reports. Asia Pac J Public Health. 2020.

9. Xu B, Gutierrez B, Mekaru S, Sewalk K, Goodwin L, Loskill A, et al. Epidemiological data from the COVID-19 outbreak, real-time case information. Sci Data. 2020;7(1):1-6.

10. Vokinger KN, Nittas V, Witt CM, Fabrikant SI, von Wyl V. Digital health and the COVID-19 epidemic: an assessment framework for apps from an epidemiological and legal perspective. Swiss Med Wkly. 2020;150(1920).

11. Gong M, Liu L, Sun X, Yang Y, Wang S, Zhu H. Cloud-Based System for Effective Surveillance and Control of COVID-19: Useful Experiences From Hubei, China. J Med Internet Res. 2020;22(4):e18948.

12. Grange ES, Neil EJ, Stoffel M, Singh AP, Tseng E, Resco-Summers K, et al. Responding to COVID-19: The UW Medicine Information Technology Services Experience. Appl Clin Inform. 2020;11(02):26575 .

13. Javanmard SH, Nasirian M, Ataei B, Vaseghi G, Vaezi A, Changiz T. Isfahan COvid-19 REgistry (I-CORE): Design and methodology. J Res Med Sci. 2020;25(1):32.

14. Talebpour M, Hadadi A, Oraii A, Ashraf H. Rationale and Design of a Registry in a Referral and Educational Medical Center in Tehran, Iran: Sina Hospital Covid-19 Registry (SHCo-19R). Adv J Emerg Med. 2020;4(2s)

15. Mounesan L, Eybpoosh S, Haghdoost A, Moradi G, Mostafavi E. Is reporting many cases of COVID-19 in Iran due to strength or weakness of Iran's health system? Iran J Microbiol. 2020;12(2):73-6.

16. Zareie B, Roshani A, Mansournia MA, Rasouli MA, Moradi G. A model for COVID-19 prediction in Iran based on China parameters. medRxiv. 2020.

17. Kazemi-Arpanahi H, Moulaei K, Shanbehzadeh M. Design and development of a web-based registry for Coronavirus (COVID-19) disease. Med J Islam Repub Iran. 2020;34(1):482-90.

18. Li J, Wang L, Guo S, Xie N, Yao L, Cao Y, et al. The Data set for Patient Information Based Algorithm to Predict Mortality Cause by COVID-19. Data Brief. 2020:105619.

19. Ashofteh A, Bravo JM. A study on the quality of novel coronavirus (COVID-19) official datasets. Stat J IAOS. (Preprint):1-11.

20. Shanbehzadeh M, Ahmadi M. Identification of the necessary data elements to report AIDS: A systematic review. Electron Physician. 2017;9(12):5920.

21. Kazemi-Arpanahi H, Vasheghani-Farahani A, Baradaran A, Mohammadzadeh N, Ghazisaeedi M. Developing a Minimum Data Set (MDS) for Cardiac Electronic Implantable Devices Implantation. Acta Inform Med. 2018;26(3):164-8.

22. Kazemi-Arpanahi H, Vasheghani-Farahani A, Baradaran A, Ghazisaeedi M, Mohammadzadeh N, Bostan H. Development of a minimum data set for cardiac electrophysiology study ablation. J Educ Health Promot. 2019;8.

23. Varghese J, Holz C, Neuhaus P, Bernardi M, Boehm A, Ganser A, et al., editors. Key Data Elements in Myeloid Leukemia. MIE; 2016.

24. Davis J, Morgans A, Burgess S. Information management for aged care provision in Australia: development of an aged care minimum dataset and strategies to improve quality and continuity of care. Health
Inf Manag. 2016:45(1):27-35.

25. Wang B, Sun Y, Duong TQ, Nguyen LD, Hanzo L. Risk-Aware Identification of Highly Suspected COVID-19 Cases in Social IoT: A Joint Graph Theory and Reinforcement Learning Approach. IEEE Access. 2020;8:115655-61.

26. Wang L, Duan Y, Zhang W, Liang J, Xu J, Zhang Y, et al. Epidemiologic and Clinical Characteristics of 26 Cases of COVID-19 Arising from Patient-to-Patient Transmission in Liaocheng, China. Clin Epidemiol. 2020;12:387.

27. Yuan J, Zou R, Zeng L, Kou S, Lan J, Li X, et al. The correlation between viral clearance and biochemical outcomes of 94 COVID-19 infected discharged patients. Inflamm Res. 2020:1-8.

28. Surveillances V. The epidemiological characteristics of an outbreak of 2019 novel coronavirus diseases (COVID-19) — China, 2020. China CDC Weekly. 2020;2(8):113-22.

29. Xu T, Chen C, Zhu Z, Cui M, Chen C, Dai H, et al. Clinical features and dynamics of viral load in imported and non-imported patients with COVID-19. Int J Infect Dis. 2020.

30. Qiu H, Wu J, Hong L, Luo Y, Song Q, Chen D. Clinical and epidemiological features of 36 children with coronavirus disease 2019 (COVID-19) in Zhejiang, China: an observational cohort study. Lancet Infect Dis. 2020.

31. Rodriguez-Morales AJ, Cardona-Ospina JA, Gutiérrez-Ocampo E, Villamizar-Peña R, Holguin-Rivera Y, Escalera-Antezana JP, et al. Clinical, laboratory and imaging features of COVID-19: A systematic review and meta-analysis. Travel Med Infect Dis. 2020:101623.

32. Li K, Wu J, Wu F, Guo D, Chen L, Fang Z, et al. The Clinical and Chest CT Features Associated with Severe and Critical COVID-19 Pneumonia. Invest Radiol. 2020.

33. Li YK, Peng S, Li LQ, Wang Q, Ping W, Zhang N, et al. Clinical and Transmission Characteristics of Covid-19-A Retrospective Study of 25 Cases from a Single Thoracic Surgery Department. Curr Med Sci. 2020:1-6.

34. Wan S, Xiang Y, Fang W, Zheng Y, Li B, Hu Y, et al. Clinical Features and Treatment of COVID-19 Patients in Northeast Chongqing. J Med Virol. 2020.

35. Jia J, Hu X, Yang F, Song X, Dong L, Zhang J, et al. Epidemiological characteristics on the clustering nature of COVID-19 in Qingdao City, 2020: a descriptive analysis. Disaster Med Public Health Prep. 2020:1-17.

36. Xia XY, Wu J, Liu HL, Xia H, Jia B, Huang WX. Epidemiological and initial clinical characteristics of patients with family aggregation of COVID-19. J Clin Virol. 2020:104360.

37. Chen J, Zhang ZZ, Chen YK, Long QX, Tian WG, Deng HJ, et al. The clinical and immunological features of pediatric COVID-19 patients in China. Genes Dis. 2020.

38. Liu K, Chen Y, Lin R, Han K. Clinical features of COVID-19 in elderly patients: A comparison with young and middle-aged patients. $\mathrm{J}$ Infect. 2020

39. Huang Y, Zhou H, Yang R, Xu Y, Feng X, Gong P. Clinical characteristics of 36 non-survivors with COVID-19 in Wuhan, China. medRxiv. 2020.

40. Tabata S, Imai K, Kawano S, Ikeda M, Kodama T, Miyoshi K, et al. The clinical characteristics of COVID-19: a retrospective analysis of 104 patients from the outbreak on board the Diamond Princess cruise ship in Japan. medRxiv. 2020.

41. Lyu P, Liu X, Zhang R, Shi L, Gao J. The performance of chest CT in evaluating the clinical severity of COVID-19 pneumonia: identifying critical cases based on CT characteristics. Invest Radiol. 2020.

42. Fu H, Xu H, Zhang N, Xu H, Li Z, Chen H, et al. Association between Clinical, Laboratory and CT Characteristics and RT-PCR Results in the Follow-up of COVID-19 patients. medRxiv. 2020.

43. Pormohammad A, Ghorbani S, Baradaran B, Khatam A, Turner R, Mansournia MA, et al. Clinical Characteristics, Laboratory Findings, Radiographic Signs and Outcomes of 52,251 Patients with Confirmed COVID-19 Infection: A Systematic Review and Meta-Analysis. Microb Pathog. 2020.

44. Mao Y, Lin W, Wen J, Chen G. Clinical and pathological characteristics of 2019 novel coronavirus disease (COVID-19): a systematic reviews. medRxiv. 2020.

45. Lomoro P, Verde F, Zerboni F, Simonetti I, Borghi C, Fachinetti C, et al. COVID-19 pneumonia manifestations atthe admission on chest ultrasound, radiographs, and CT: single-center study and comprehensive radiologic literature review. Eur J Radiol Open. 
$2020 \cdot 100231$

46. Adhikari SP, Meng S, Wu YJ, Mao YP, Ye RX, Wang QZ, et al. Epidemiology, causes, clinical manifestation and diagnosis, prevention and control of coronavirus disease (COVID-19) during the early outbreak period: a scoping review. Infect Dis Poverty. 2020;9(1):1-12.

47. Chang $\mathrm{TH}, \mathrm{Wu}$ JL, Chang LY. Clinical characteristics and diagnostic challenges of pediatric COVID-19: A systematic review and meta-analysis. J Formos Med Assoc. 2020.

48. Wang Y, Wang Y, Chen Y, Qin Q. Unique epidemiological and clinical features of the emerging 2019 novel coronavirus pneumonia (COVID-19) implicate special control measures. J Med Virol. 2020;92(6):568-76.

49. Hassan S, Sheikh FN, Jamal S, Ezeh JK, Akhtar A. Coronavirus (COVID-19): a review of clinical features, diagnosis, and treatment. Cureus. 2020;12(3)

50. Siordia Jr JA. Epidemiology and Clinical Features of COVID-19: A Review of Current Literature. J Clin Virol. 2020:104357.

51. Zhang G, Hu C, Luo L, Fang F, Chen Y, Li J, et al. Clinical features and short-term outcomes of 221 patients with COVID-19 in Wuhan, China. J Clin Virol. 2020:104364.

52. Fu L, Wang B, Yuan T, Chen X, Ao Y, Fitzpatrick T, et al. Clinical characteristics of coronavirus disease 2019 (COVID-19) in China: a systematic review and meta-analysis. J Infect. 2020.

53. Lovato A, de Filippis C. Clinical Presentation of COVID-19: A Systematic Review Focusing on Upper Airway Symptoms. Entechnology. 2020:0145561320920762.

54. Zhao X, Zhang B, Li P, Ma C, Gu J, Hou P, et al. Incidence, clinical characteristics and prognostic factor of patients with COVID-19: a systematic review and meta-analysis. medRxiv. 2020.

55. Fang Z, Yi F, Wu K, Lai K, Sun X, Zhong N, et al. Clinical Characteristics of 2019 Coronavirus Pneumonia (COVID-19): An Updated Systematic Review. medRxiv. 2020.

56. Zheng Y, Xu H, Yang M, Zeng Y, Chen H, Liu R, et al. Epidemiological Characteristics and Clinical Features of 32 Critical and 67 Noncritical Cases of COVID-19 in Chengdu. J Clin Virol. 2020:104366.

57. Wang X, Fang J, Zhu Y, Chen L, Ding F, Zhou R, et al. Clinical characteristics of non-critically ill patients with novel coronavirus infection (COVID-19) in a Fangcang Hospital. Clin Microbiol Infect. 2020.

58. Zhao W, Zhong Z, Xie X, Yu Q, Liu J. CT scans of patients with 2019 novel coronavirus (COVID-19) pneumonia. Theranostics. 2020;10(10):4606.

59. Zhu Y, Gao ZH, Liu YL, Xu DY, Guan TM, Li ZP, et al. Clinical and CT imaging features of 2019 novel coronavirus disease (COVID19). J Infect. 2020.

60. Davey CJ, Slade SV, Shickle D. A proposed minimum data set for international primary care optometry: a modified Delphi study. Ophthalmic Physiol Opt. 2017;37(4):428-39.

61. Hashemi N, Sheikhtaheri A, Hashemi N, Rawassizadeh R. Electronic Medical Records for Mental Disorders: What Data Elements Should These Systems Contain? Stud Health Technol Inform. 2019;260:25-32.

62. Shaghaghi A, Bhopal RS, Sheikh A. Approaches to recruiting 'hardto-reach'populations into research: a review of the literature. Health Promot Perspect. 2011;1(2):86.

63. Sadler GR, Lee HC, Lim RSH, Fullerton J. Recruitment of hard-toreach population subgroups via adaptations of the snowball sampling strategy. Nurs Health Sci. 2010;12(3):369-74.

64. Lapinlampi N, Melin E, Aronica E, Bankstahl JP, Becker A, Bernard $\mathrm{C}$, et al. Common data elements and data management: Remedy to cure underpowered preclinical studies. Epilepsy Res. 2017;129:87-90.

65. Grinnon ST, Miller K, Marler JR, Lu Y, Stout A, Odenkirchen J, et al. National institute of neurological disorders and stroke outcome set for hip fracture trials. Bone Joint J. 2014;96(8):1016-23.

66. Wilkinson MD, Dumontier M, Aalbersberg IJ, Appleton G, Axton M, Baak A, et al. The FAIR Guiding Principles for scientific data management and stewardship. Sci Data. 2016;3.

67. Haywood K, Griffin X, Achten J, Costa M. Developing a core common data element project-approach and methods. Clin Trials J. 2012;9(3):322-9.

68. Lutomski JE, Baars MAE, Schalk BWM, Boter H, Buurman BM, den Elzen WPJ, et al. The development of the Older Persons and Informal Caregivers Survey Minimum DataSet (TOPICS-MDS): a large-scale data sharing initiative. PLoS One. 2013;8(12):e81673-e. 69. Riley WT, Glasgow RE, Etheredge L, Abernethy AP. Rapid responsive, relevant (R3) research: a call for a rapid learning health research enterprise. Clin Transl Med. 2013;2(1):10. 